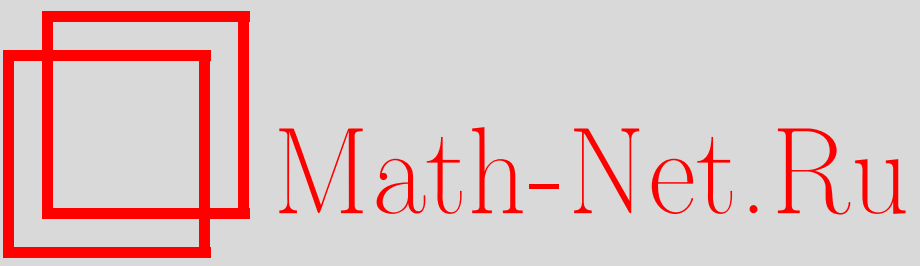

М. Е. Чанга, О количестве простых, дающих бесквадратные суммы с заданными числами, УМН, 2003, том 58, выпуск 3, 197-198

DOI: https://doi.org/10.4213/rm637

Использование Общероссийского математического портала Math-Net.Ru подразумевает, что вы прочитали и согласны с пользовательским соглашением

http://www.mathnet.ru/rus/agreement

Параметры загрузки:

IP: 3.85 .5 .30

26 апреля 2023 г., 18:16:18 


\title{
О КОЛИЧЕСТВЕ ПРОСТЫХ, ДАЮЩИХ БЕСКВАДРАТНЫЕ СУММЫ С ЗАДАННЫМИ ЧИСЛАМИ
}

\author{
М. Е. ЧАНГА
}

Пусть даны $k$ целштх чисел $l_{i}$ с условием $0 \leqslant l_{1}<l_{2}<\cdots<l_{k}$. Можно поставить такой вопрос: как много натуральных чисел $n \leqslant x$ таких, что все числа $n+l_{1}, \ldots, n+l_{k}$ одновременно свободны от $m$-х степеней? Если через $\delta_{m}(n)$ обозначить характеристическую функцию множества натуральных чисел, свободных от $m$-х степеней, то эта задача сведется к изучению суммш

$$
S(x)=\sum_{n \leqslant x} \delta_{m}\left(n+l_{1}\right) \cdots \delta_{m}\left(n+l_{k}\right)
$$

В конце сороковых годов Л. Мирский [1], [2] получил для этой суммы асимптотическую формулу.

Теорема (Л. Мирский). Пусть $\alpha(n)$ обозначает количество чисел $l_{i}$, различных по модулю . Тогда

$$
S(x)=x \prod_{p}\left(1-\frac{\alpha\left(p^{m}\right)}{p^{m}}\right)+O\left(x^{\frac{2}{m+1}+\varepsilon}\right) .
$$

Доказательство этой теоремы основано на известной формуле для функции $\delta_{m}(n)$

$$
\delta_{m}(n)=\sum_{d^{m} \mid n} \mu(d) .
$$

Представить константу в главном члене в виде произведения по простьм можно с помощью многомерного аналога тождества Эйлера или косвенного подхода, использующего решето. Эта теорема позволяет заключить: $S(x)$ неограниченна тогда и только тогда, когда числа $l_{i}$ не покрьвают полной системы вычетов ни по какому $p^{m}$.

В настоящей работе рассматривается задача о количестве простых чисел $p \leqslant x$ таких, что $p+l_{1}, \ldots, p+l_{k}$ одновременно свободны от $m$-х степеней. Введем обозначение

$$
T(x)=\sum_{p \leqslant x} \delta_{m}\left(p+l_{1}\right) \cdots \delta_{m}\left(p+l_{k}\right) .
$$

Тогда справедлива следующая теорема.

Теорема 1. Пусть $\beta(n)$ обозначает количество чисел $l_{i}$, pазличных по модулю $n u$ взаимно простых с ним. Тогда

$$
T(x)=\pi(x) \prod_{p}\left(1-\frac{\beta\left(p^{m}\right)}{\varphi\left(p^{m}\right)}\right)+O\left(\frac{x}{\log ^{A} x}\right),
$$

где $A$ - произвольное положительное число.

Доказательство этой теоремы исполњзует теорему Зигеля-Валњфиша и, в основном, следует методу Мирского. Однако способ, примененньй им при оценке остатка, здесь недостаточен. В этом случае остаток удается оценить, последовательно применяя формулу (1) и анализируя получающиеся на каждом шаге соотношения. Как и прежде, найденная асимптотическая формула дает следствие: $T(x)$ неограниченна тогда и только тогда, когда числа $l_{i}$ не покрьвают приведенной системы вычетов ни по какому $p^{m}$.

Пусть теперь задано нецелое число $a$, большее единицы. Тогда натуральное число $D$ и целое число $r$ с условием $0 \leqslant r<D$ определяют множество

и сумму

$$
E=\bigcup_{n=0}^{\infty}\left[(D n+r)^{1 / a},(D n+r+1)^{1 / a}\right)
$$

$$
U(x)=\sum_{\substack{p \leqslant x \\ p \in E}} \delta_{m}\left(p+l_{1}\right) \cdots \delta_{m}\left(p+l_{k}\right) .
$$

В этом случае справедлива следующая теорема. 
Теорема 2. Пусть $\beta(n)$ обозначает количество чисел $l_{i}$, различных по модулю $n$ и взаимно простых с ним. Тогда

$$
U(x)=\frac{\pi(x)}{D} \prod_{p}\left(1-\frac{\beta\left(p^{m}\right)}{\varphi\left(p^{m}\right)}\right)+O\left(\frac{x}{\log ^{A} x}\right),
$$

где $D \leqslant \log ^{A} x, A-$ произвольное положительное число.

Доказательство дословно повторяет доказательство теоремы 1 , но использует теорему Зигеля-Вальфиша для простых из множества $E$. Последняя является следствием теоремы об асимптотическом поведении тригонометрических сумм с простыми числами из множества $E$ [3].

\section{СПИСОК ЛИТЕРАТУРЫ}

[1] L. Mirsky // Proc. London Math. Soc. (2). 1949. V. 50. P. 497-508. [2] L. Mirsky // Quart. J. Math. Oxford Ser. 1947. V. 18. № 71. P. 178-182. [3] M. Е. Чанга // Матем. заметки. 2003. T. 73. № 3. C. 423-436.

Математический институт

им. В.А. Стеклова РАН

Принято редколлегией

24.03.2003 